\title{
REVIEW
}

\section{Pulse Oximetry Monitoring of Infants Who Require Prone Position For Sleep - Is There Evidence That This Practice Reduces SIDS?}

\author{
Nicole Alcolado', Daniel M Hughes MD FRCPC ${ }^{2}$ \\ ${ }^{1}$ Class of 2014, Faculty of Medicine, Dalhousie University \\ ${ }^{2}$ Pediatric Respiratory Medicine, IWK Health Centre, Halifax, Nova Scotia, Canada
}

\begin{abstract}
At the IWK Health Centre infants with special medical or developmental needs who require prone and/or side-lying positions for sleep are currently monitored by pulse oximetry to presumably reduce the risk of SIDS. The aim of this paper was to determine if there is any evidence in the literature demonstrating that pulse oximetry monitoring of infants who sleep in the prone position has any effect on mortality from SIDS. A review of the literature was conducted and no studies were found that specifically address this question. Since medical practices should be based on evidence and there is currently none for this protocol, we believe it may be doing more harm than good.
\end{abstract}

Sudden Infant Death Syndrome (SIDS), defined as the sudden death of an infant before one year of age that remains unexplained following a clinical history, examination of the death scene, and autopsy, ${ }^{1-3}$ is the leading cause of post-neonatal infant death in the developed world. ${ }^{4,5}$ SIDS most commonly occurs in the time period between one and six months of age. ${ }^{6,7}$ Although the cause of SIDS is still unknown and believed to be multifactorial, the 'Triple Risk' hypothesis, which was first proposed almost 20 years ago, is the most widely accepted model to explain its pathophysiology. ${ }^{8}$ In this model SIDS results from the presence of three simultaneous risk factors: 1 ) a vulnerable infant (such as prematurity or low birth weight), 2) a critical period during the development of homeostatic control mechanisms, and 3) an exogenous stressor. ${ }^{8}$ There is an abundance of evidence suggesting that sleep in the prone as opposed to the supine position is one of the major exogenous stressors associated with SIDS.9-14 Since 1992 the American Academy of Pediatrics (AAP) has recommended that infants be placed in the supine position for sleep in order to reduce the risk of SIDS, ${ }^{2}$ and implementation of the 'Back to Sleep' program has reduced the incidence of SIDS deaths by over $50 \%$ worldwide. ${ }^{15}$ In 1992 the rate of SIDS was 1.2/1,000 live births and only $13 \%$ of infants slept on their backs, whereas in 2006 the rate of SIDS dropped to 0.55/1,000 live births and more than $75 \%$ of infants slept supine. ${ }^{15}$

There is still some debate as to whether prone position is superior to supine in infants with gastro-esophageal reflux disease (GERD) ${ }^{16-18}$ Current AAP guidelines recommend that prone sleep position be considered in special cases where the risk of complications from
GERD is greater than the potential increased risk of SIDS. ${ }^{19}$ In 2006, the IWK Health Centre's Policy on Infant Positioning stated in its protocol for infants with special medical or developmental needs who require prone and/or side-lying positions for sleep, electronic monitoring is required. ${ }^{20}$ Electronic monitoring is done by pulse oximetry $(\mathrm{SpO} 2)$, which is a non-invasive way to monitor oxygen-hemoglobin saturation. ${ }^{21}$

We believe that this policy of monitoring otherwise healthy infants, that is those who are not vulnerable to SIDS and who are put to sleep in the prone position, may be problematic for several reasons. First, practice guidelines should be evidence-based, and we are not aware of any evidence demonstrating that pulse oximetry monitoring, of otherwise healthy infants in the hospital, has been shown to reduce their risk of SIDS. Furthermore, pulse oximetry averages the signal obtained over several seconds. This may delay the detection of acute changes in oxygenation by several seconds, consequently delaying the detection of acute hypoxemia. ${ }^{22}$ Finally, once these children are discharged from the hospital and will no longer be electronically monitored but still sleeping in the prone position, their parents may be afraid that they will die from SIDS because they are not being monitored.

The purpose of this paper was to determine if there is any evidence in the literature demonstrating that pulse oximetry monitoring of otherwise healthy infants who require prone sleep position reduces SIDS deaths. In the event there is no evidence, this practice of monitoring may be doing more harm than good, as it may be creating unnecessary parental anxiety. 


\section{Methods}

All 10 papers cited in the IWK Health Centre's policy ${ }^{20}$ were reviewed for evidence that pulse oximetry monitoring of prone infants reduces the incidence of SIDS deaths. None of the citations included any evidence specifically addressing whether electronic monitoring of infants in the prone position for sleep reduced incidence of SIDS. ${ }^{1,4,5,23-29}$

The goal was then to determine if there is evidence in the literature specifically addressing the question: does pulse oximetry monitoring of otherwise healthy infants (i.e. those not at increased risk for SIDS) put to sleep in the prone position reduce the incidence of SIDS? The population of interest was infants sleeping in the prone position. The comparison was pulse oximetry monitoring versus no pulse oximetry monitoring, and the outcome was SIDS deaths.

A literature review was conducted using both the PubMed Clinical Queries and the PubMed database tools (from Jan 1966 - Dec 2011). In the PubMed Clinical Queries database, each available search category (etiology, diagnosis, therapy, prognosis, clinical prediction guides) was used with a broad search scope. The search strategy: 'infant AND prone AND pulse oximetry monitoring AND SIDS' yielded two clinical studies results..$^{30,31}$ Removing infant from the search but still including: 'pulse oximetry monitoring AND prone AND SIDS' yielded only the same two papers as before. ${ }^{30,31}$ The second search: 'infant AND prone AND pulse oximet" AND SIDS' resulted in three papers. ${ }^{30,32,33}$ Removing infant from the search for 'prone AND pulse oximet" AND SIDS' yielded the same three results. ${ }^{30,32,33}$ Expanding the search to the general PubMed database (not limited to the Clinical Queries section) with the limit of infant (age birth - 23 months) and the search strategy: 'pulse oximetry AND prone AND SIDS' yielded five papers..$^{30,31,33-35}$ In summary, a total of six unique papers were identified ${ }^{30-35}$ from the literature search which could possibly address the question.

None of the papers identified in the literature search addressed the specific research question. The Sahni et al. ${ }^{30}$ study examined low birth weight (LBW) and the Saiki et al. ${ }^{34}$ and Poets et al. ${ }^{32}$ studies examined premature babies, both of which are populations at increased risk of SIDS ${ }^{8}$ and therefore not relevant to our population of interest. The Poets et al. study from $1995^{33}$ also included babies at increased risk of SIDS, either because they were premature, had a sibling who died of SIDS, or had a suspected airway obstruction. Furthermore, Poets et al. found no significant differences in oxygen desaturations measured ${ }^{32}$ while
Saiki et al ${ }^{34}$ found no significant differences in oxygen saturation levels between prone or supine position lying infants. The Skadburg et al. ${ }^{31}$ study measured oxygen saturation in full term infants who were not at increased risk for SIDS, hence the criteria of our population of interest was met, but the purpose of the study was very specific and not relevant to our search. It was to measure if side-sleeping infants tilted their face to the side when moved to prone position. The testing in this study was terminated once infants had spent three minutes face down, and SIDS outcomes were not directly measured. The Uchigasaki et al. study was only available in Japanese so could not be interpreted. ${ }^{35}$ However, information extracted from the abstract revealed that it is only a report on two infants, hence of limited value to the purpose of this literature search.

\section{Discussion}

There is an abundance of evidence demonstrating that infants placed in the supine as opposed to the prone position for sleep have a decreased incidence of SIDS.9-14 At the IWK Health Centre, infants with special medical or developmental needs who require prone lying position for sleep are currently monitored by pulse oximetry in accordance with the Infant Positioning Policy. ${ }^{20}$ The purpose of this paper was to determine if there is any evidence in the literature demonstrating that pulse oximetry monitoring of otherwise healthy infants who require prone sleep position reduces mortality from SIDS. In this literature review, no journal articles were found which directly addressed either our population of interest or our expected outcome of SIDS deaths. We have therefore found no evidence to suggest that electronic monitoring of infants who sleep in the prone position for special considerations has any impact on the incidence of SIDS.

Aside from the lack of evidence behind this protocol, we believe it may also be causing undue distress to parents. Studies show that parent practices at home are strongly influenced by hospital nursery practices when it comes to the topic of SIDS. ${ }^{27,36,37}$ Therefore, altering an infant's sleep regimen by removing one component from it, pulse oximetry monitoring, may be unsettling to parents and cause unnecessary anxiety. Since medical protocols should be based in evidence, and there is no evidence behind this protocol, there should be no need to monitor these otherwise healthy infants. The newer generations of pulse oximeters are more accurate but pulse oximetry readings may be unreliable during periods of infant movement. ${ }^{38-40}$ They may miss drops in oxygenation if they occur in the absence of bradycardia or central apnea, ${ }^{21,41}$ and they are known to produce false alarms. ${ }^{21,38,39,41}$ 
It should be noted that simply because there is no available evidence demonstrating that electronic monitoring of otherwise healthy infants has any impact on preventing mortality from SIDS does not necessarily mean that this is so. There is a need for further research on this subject including a review of policies in other hospitals. The lack of evidence behind this protocol suggests that it should be discontinued.

\section{Conclusions}

There should be a purpose to each medical intervention and protocols should be based on evidence. In this paper we have concluded that there is currently no evidence demonstrating that the pulse oximetry monitoring of non-vulnerable infants, lying prone for sleep, reduces mortality from SIDS. Until evidence shows otherwise, we believe that the policy of monitoring these infants at the IWK Health Centre may be doing more harm than good.

\section{References}

1. Health Canada SIDS Sudden Infant Death Syndrome Joint Statement: Back to Sleep. 2011. http://www.hc-sc.gc.ca/hppb/ childhood-youth/cyfh/sids/joint_statement.html updated 2011-06-13. (Accessed Dec, 2011).

2. Changing concepts of sudden infant death syndrome: implications for infant sleeping environment and sleep position. American Academy of Pediatrics. Task Force on Infant Sleep Position and Sudden Infant Death Syndrome. Pediatrics 2000;105(3):650-656.

3. Willinger M, James LS, Catz C. Defining the sudden infant death syndrome (SIDS): deliberations of an expert panel convened by the National Institute of Child Health and Human Development Pediatr Pathol 1991;11(5):677-684.

4. American Academy of Pediatrics Task Force on Sudden Infant Death Syndrome. The changing concept of sudden infant death syndrome: diagnostic coding shifts, controversies regarding the sleeping environment, and new variables to consider in reducing risk. Pediatrics 2005;116(5):1245-1255.

5. Galland BC, Taylor BJ, Bolton DP. Prone versus supine sleep position: a review of the physiological studies in SIDS research. J Paediatr Child Health 2002;38(4):332-338.

6. Blair PS, Sidebotham P, Berry PJ, Evans M, Fleming PJ. Major epidemiological changes in sudden infant death syndrome: a 20-year population-based study in the UK. Lancet 2006 Jan;367(9507):314-9.

7. Leach CE, Blair PS, Fleming PJ, Smith IJ, Platt MW, Berry PJ, Golding J. Epidemiology of SIDS and explained sudden infant deaths. CESDI SUDI Research Group. Pediatrics 1999;104(4):e43.

8. Guntheroth WG, Spiers PS. The triple risk hypotheses in sudden infant death syndrome. Pediatrics 2002;110(5):e64.

9. Carpenter RG, Irgens LM, Blair PS, England PD, Fleming P, Huber J, Jorch G, Schreuder P. Sudden unexplained infant death in 20 regions in Europe: case control study. Lancet 2004;363(9404):185 191.

10. Li DK, Petitti DB, Willinger M, McMahon R, Odouli R, Vu H, Hoffman HJ. Infant sleeping position and the risk of sudden infant death syndrome in California, 1997-2000. Am J Epidemiol 2003;157(5):446-455.
11. Gessner BD, Ives GC, Perham-Hester KA. Association between sudden infant death syndrome and prone sleep position, bed sharing, and sleeping outside an infant crib in Alaska. Pediatrics 2001;108(4):923-927.

12. Brooke H, Gibson A, Tappin D, Brown H. Case-control study of sudden infant death syndrome in Scotland, 1992-5. BMJ 1997 May 24;314(7093):1516-1520.

13. Mitchell EA, Tuohy PG, Brunt JM, Thompson JM, Clements MS, Stewart AW, Ford RP, Taylor BJ. Risk factors for sudden infant death syndrome following the prevention campaign in New Zealand: a prospective study. Pediatrics 1997;100(5):835-840.

14. Oyen N, Markestad T, Skaerven R, Irgens LM, Helweg-Larsen $\mathrm{K}$, Alm B, Norvenius G, Wennergren G. Combined effects of sleeping position and prenatal risk factors in sudden infant death syndrome: the Nordic Epidemiological SIDS Study. Pediatrics 1997;100(4):613-621.

15. 'Back to Sleep Public Education Campaign' NIH, 2011. http://www. nichd.nih.gov/sids/ Update - 10/18/2011. (Accessed Dec, 2011).

16. Hunt CE, Lesko SM, Vezina RM, McCoy R, Corwin MJ, Mandell F, Willinger M, Hoffman HJ, Mitchell AA. Infant sleep position and associated health outcomes. Arch Pediatr Adolesc Med 2003;157(5):469-474.

17. Vandenplas Y, Hauser B.Gastro-oesophageal reflux, sleep pattern, apparent life threatening event and sudden infant death. The point of view of a gastro-enterologist.Eur J Pediatr 2000;159(10):726-729.

18. Hunt L, Fleming P, Golding J. Does the supine sleeping position have any adverse effects on the child? I. Health in the first six months. The ALSPAC Study Team. Pediatrics 1997;100(1):E11.

19. American Academy of Pediatrics. 'Guidelines for Evaluation and Treatment of Gastroensophageal Reflux in Infants and Children'. 2001. http://aappolicy.aappublications.org/misc/Guidelines_ for_Evaluation_and_Treatment_of_Gastroesophageal_Reflux. dtl. (Accessed Dec, 2011).

20. IWK Health Centre Clinical Policy/Objective manual. 2006. Policy 1110 - Infant Positioning. Last Published 1/17/2006.

21. Gélinas JF, Davis GM, Arlegui C, Côté A. Prolonged, documented home-monitoring of oxygenation in infants and children. Pediatr Pulmonol 2008; 43(3):288-296.

22. Stoneham MD. Uses and limitations of pulse oximetry. Br J Hosp Med; 54(1):35-41.

23. Dubé K, Flake ML. Early prevention. Occipital flattening of positional origin. Can Nurse 2003;99(1):16-21.

24. Neufeld, S. \& Birkett, S. What to do about flat heads: Preventing and treating positional occipital flattening. Axone 2000;22(2):29 -31.

25. Lockridge T, Taquino LT, Knight A. Back to sleep: is there room in that crib for both AAP recommendations and developmentally supportive care? Neonatal Netw 1999;18(5):29-33.

26. Reproductive Care Program of Nova Scotia (2009) RCP Endorsement: Back to Sleep National Guidelines. 2009. http:// rcp.nshealth.ca/sites/default/files/clinical-practice-guidelines/ Back_to_Sleep_Sep2009.pdf. (Accessed Dec, 2011).

27. Colson ER, Bergman DM, Shapiro E, Leventhal JH. Position for newborn sleep: associations with parents' perceptions of their nursery experience. Birth 2001;28(4):249-253.

28. National Institute of Child Health \& Human Development. Health Information \& Media - SIDS: "Back to Sleep" Campaign Babies sleep safest on their backs: Reduce the risk of Sudden Infant Death Syndrome. NIH, 2006. http://www.nichd.nih.gov/sids/ reduce_infant_risk Modified 2006 to: http://www.nichd.nih.gov/ publications/pubs/safe_sleep_gen.cfm. (Accessed Dec, 2011).

29. Persing J, James H, Swanson J, Kattwinkel J; American Academy of Pediatrics Committee on Practice and Ambulatory Medicine, Section on Plastic Surgery and Section on Neurological Surgery. Prevention and management of positional skull deformities in 
infants. American Academy of Pediatrics Committee on Practice and Ambulatory Medicine, Section on Plastic Surgery and Section on Neurological Surgery. Pediatrics 2003;112(1 Pt 1):199-202.

30. Sahni R, Schulze KF, Ohira-Kist K, Kashyap S, Myers MM, Fifer WP. Interactions among peripheral perfusion, cardiac activity, oxygen saturation, thermal profile and body position in growing low birth weight infants. Acta Paediatr 2010;99(1):135-139.

31. Skadberg BT, Markestad T. Infant behaviour in response to a change in body position from side to prone during sleep.Eur J Pediatr 1996;155(12):1052-1056.

32. Poets CF, Bodman A. [Sleeping position for preterm infants]. Z Geburtshilfe Neonatol 2008;212(1):27-29.

33. Poets CF, Rudolph A, Neuber K, Buch U, von der Hardt $\mathrm{H}$. Arterial oxygen saturation in infants at risk of sudden death: influence of sleeping position. Acta Paediatr 1995;84(4):379382.

34. Saiki T, Rao H, Landolfo F, Smith AP, Hannam S, Rafferty GF, Greenough A. Sleeping position, oxygenation and lung function in prematurely born infants studied post term. Arch Dis Child Fetal Neonatal Ed 2009;94(2):F133-F137.

35. Uchigasaki S, Mukai T, Yamaguchi N, Tsukamoto S, Oshida S, Sato Y, Suzuki T. [Arterial oxygen saturation monitoring of sleeping infants by pulse-oximeter--effect of sleeping position on arterial oxygen saturation in infants]. Nihon Hoigaku Zasshi 1995;49(2):87-91.

36. Rao H, May C, Hannam S, Rafferty GF, Greenough A. Survey of sleeping position recommendations for prematurely born infants on neonatal intensive care unit discharge. Eur J Pediat. 2007;166(8):809-811.

37. Colson ER, Joslin SC. Changing nursery practice gets inner-city infants in the supine position for sleep. Arch Pediatr Adolesc Med 2002;156(7):717-720.

\section{MAKE THE RIGHT CALL}

For the best selection of reference books and materials, call the Health Sciences Bookstore.

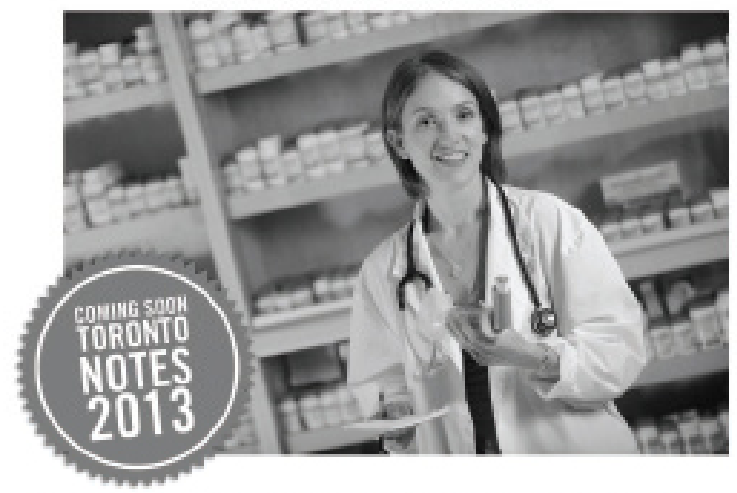

The Dalhousie Health Sciences Bookstore carries a wide selection of text and reference books in addition to scrubs, medical supplies, gifts, and more.

From school to practice, weire always an call for you. Come by or visit our website today.

Visit Us at:

Dentistry Building

Hesith Stiences Bookstare

5981 University Avenas

Tal 902.494 .360

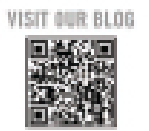

38. Lawless ST. Crying wolf: false alarms in a pediatric intensive care unit. Crit Care Med 1994;22(6):981-985.

39. Poets CF, Southall DP. Noninvasive monitoring of oxygenation in infants and children: practical considerations and areas of concern. Pediatrics 1994;93(5):737-746.

40. Meyts I, Reempts PV, Boeck KD. Monitoring of haemoglobin oxygen saturation in healthy infants using a new generation pulse oximeter which takes motion artifacts into account. Eur J Pediatr. 2002;161(12):653-655.

41. Bohnhorst B, Peter CS, Poets CF. Pulse oximeters' reliability in detecting hypoxemia and bradycardia: comparison between a conventional and two new generation oximeters. Crit Care Med 2000;28(5):1565-1568.
Looking for a great place to live and work? Cumberland County Nowa Scotia is rich in spectacular scenery and natural resources, a wealth of cultural opportunities and most importantly, great pecple. In Cumberland County you are never more than minutes from the sea and we offer a great blend of recreational opportunities like sking, golf, hiking, saling and much more. Combine that with easy access to eductiona and culural activilies, urban centers and travel routes, then add a dash of our country's special traeks like lobster, blueberries and maple sugar and you have the formula for perfect work-life balance. If's al here waiting for you in Cumberland County.

The Cumberland Health Authority (CHA) provides health care services to this northem-most part of Nova Scotia. The Districts services range from acule to long-tem care and include public health, addiction. mental health and primary care programs. From five health care facilifes and several community-based sites, a total stafing complement of nearly 800 health professionals and support staff provide quality care to Cumberland Countys 33,000 elizens.

We have physician opportunifes for:

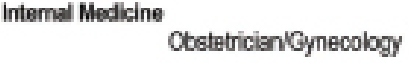

www.cha.nshealth.ca Ph: 902-661-1090

\section{Palliative Mediche}

WWW.dal.ca/tookston 